\title{
WEIGHTED WEAK-TYPE INEQUALITIES FOR GENERALIZED HARDY OPERATORS
}

\author{
A. L. BERNARDIS, F. J. MARTÍN-REYES, AND P. ORTEGA SALVADOR
}

Received 13 June 2006; Accepted 21 September 2006

We characterize the pairs of weights $(v, w)$ for which the Hardy-Steklov-type operator $T f(x)=g(x) \int_{s(x)}^{h(x)} K(x, y) f(y) d y$ applies $L^{p}(v)$ into weak- $L^{q}(w), q<p$, assuming certain monotonicity conditions on $g, s, h$, and $K$.

Copyright (c) 2006 A. L. Bernardis et al. This is an open access article distributed under the Creative Commons Attribution License, which permits unrestricted use, distribution, and reproduction in any medium, provided the original work is properly cited.

\section{Introduction}

Let us consider the Hardy-Steklov-type operator defined by

$$
T f(x)=g(x) \int_{s(x)}^{h(x)} K(x, y) f(y) d y, \quad f \geq 0,
$$

where $g$ is a nonnegative measurable function, $s$ and $h$ are continuous and increasing functions $(x<y \Rightarrow s(x) \leq s(y), h(x) \leq h(y))$ defined on an interval $(a, b)$ such that $s(x) \leq$ $h(x)$ for all $x \in(a, b)$, and the kernel $K(x, y)$ defined on $\{(x, y): x \in(a, b)$ and $s(x) \leq y \leq$ $h(x)\}$ satisfies

(i) $K(x, y) \geq 0$,

(ii) it is increasing and continuous in $x$ and decreasing in $y$,

(iii) $K(x, z) \leq D[K(x, h(y))+K(y, z)]$ for $y \leq x$ and $s(x) \leq z \leq h(y)$, where the constant $D>1$ is independent of $x, y$, and $z$.

Gogatishvili and Lang [3] characterized the pairs of weights for the strong- and weaktype $(p, q)$ inequalities for the operator $T$ in the case $p \leq q$. Actually, in [3] the authors deal with Banach functions spaces with some extra condition. On the other hand, Chen and Sinnamon [2] have characterized the weighted strong-type inequality for $1<p$, $q<\infty$ in terms of a normalizing measure. In both papers, they work with more general functions $s, h$, and $K$. 
The goal of this paper is to characterize the weighted weak-type inequalities in the case $q<p$. It is well known that strong-type inequalities for the operator $T$ can be deduced directly from the corresponding ones for $g(x)=1$, but this is not the case when we work with weak-type inequalities. In [5] it was characterized the weighted weak-type inequality in the case $q<p$ for the operator $T$ when $s \equiv 0, h(x)=x$, and $K \equiv 1$. The result was obtained for monotone functions $g$. In fact, in the proof of the result the authors used the condition

$$
\inf _{x \in E} g(x)=\inf _{x \in(\alpha, \beta)} g(x)
$$

for any bounded set $E$, where $\alpha=\inf E$ and $\beta=\sup E$. This property clearly holds if $g$ is monotone or if there exists $x_{0}$ such that $g$ is increasing in $\left(a, x_{0}\right]$ and decreasing in $\left[x_{0}, b\right)$. In our result, we will assume (1.2) and the same condition for the function $g(x) K(x, y)$, that is, for all $y$ and every bounded set $E_{y} \subset\{x: s(x) \leq y \leq h(x)\}$,

$$
\inf _{x \in E_{y}}[g(x) K(x, y)]=\inf _{x \in\left(\alpha_{y}, \beta_{y}\right)}[g(x) K(x, y)]
$$

where $\alpha_{y}=\inf E_{y}$ and $\beta_{y}=\sup E_{y}$.

Examples of Hardy-Steklov-type operators are the modified Riemann-Liouville operators defined for $\alpha>0$ and $\eta \in \mathbb{R}$ as $x^{\eta} \int_{0}^{x}(x-y)^{\alpha} f(y) d y$ or the more general version $x^{\eta} \int_{A x}^{B x}(x-y)^{\alpha} f(y) d y$, with $0<A<B \leq 1$ and $x>0$; the modified logarithmic kernel operators $x^{\eta} \int_{0}^{x} \log ^{\beta}(x / y) f(y) d y$, with $\beta>0$ and $\eta \in \mathbb{R}$; the Steklov operator $T f(x)=\int_{x-1}^{x+1} f$; and the Riemann-Liouville operators, with general variable limits $\int_{s(x)}^{h(x)}(x-y)^{\alpha} f(y) d y$, with $s(x) \leq h(x) \leq x$. This last operator was studied in [6] in the case $-1<\alpha<0$.

As far as we know, our result is new even for the particular cases $T f(x)=g(x) \int_{0}^{x} K(x$, $y) f(y) d y$ and $T f(x)=\int_{s(x)}^{h(x)} K(x, y) f(y) d y$. For this last operator, conditions (1.2) and (1.3) hold trivially because $K(x, y)$ is increasing in $x$.

The notation is standard: $w(E)$ denotes the integral $\int_{E} w$; if $1<p<\infty$, then $p^{\prime}$ denotes the conjugate exponent of $p$ defined by $1 / p+1 / p^{\prime}=1$, and $L^{q, \infty}(w)$ will denote the space of measurable functions $f$ such that

$$
\|f\|_{q, \infty ; w}=\sup _{\lambda>0} \lambda(w(\{x:|f(x)|>\lambda\}))^{1 / q}<\infty .
$$

\section{Statement and proof of the result}

In the next theorem we state the result of this article.

THeOREM 2.1. Let $s$ and $h$ be increasing continuous functions defined on an interval $(a, b)$ satisfying $s(x) \leq h(x)$ for $x \in(a, b)$. Let $K(x, y)$ be defined on $\{(x, y): x \in(a, b)$ and $s(x) \leq$ $y \leq h(x)\}$ satisfying (i), (ii), (iii) and let $g$ be a nonnegative function defined on $(a, b)$ satisfying (1.2) and (1.3). Let $q$, $p$, and $r$ be such that $0<q<p, 1<p<\infty$, and $1 / r=1 / q-1 / p$. 
A. L. Bernardis et al. 3

Let $w$ and $v$ be nonnegative measurable functions defined on $(a, b)$ and $(s(a), h(b))$, respectively. The following statements are equivalent.

(i) There exists a positive constant $C$ such that

$$
[w(\{x \in(a, b): T f(x)>\lambda\})]^{1 / q} \leq \frac{C}{\lambda}\left(\int_{s(a)}^{h(b)} f^{p} v\right)^{1 / p}
$$

for all $f \geq 0$ and all positive real number $\lambda$.

(ii) The functions

$$
\Phi_{1}(x)=\sup \left\{\inf _{t \in(c, d)}[g(t) K(t, h(\bar{c}))]\left(\int_{c}^{d} w\right)^{1 / p}\left(\int_{s(d)}^{h(\bar{c})} v^{1-p^{\prime}}\right)^{1 / p^{\prime}}\right\},
$$

where the supremum is taken over all the numbers $\bar{c}, c$, and $d$ such that $a \leq \bar{c} \leq c<x<d \leq b$ and $s(d) \leq h(\bar{c})$ and

$$
\Phi_{2}(x)=\sup \left\{\left(\inf _{t \in(c, d)} g(t)\right)\left(\int_{c}^{d} w\right)^{1 / p}\left(\int_{s(d)}^{h(c)} K^{p^{\prime}}(c, y) v^{1-p^{\prime}}(y) d y\right)^{1 / p^{\prime}}\right\},
$$

where the supremum is taken over all the numbers $c$ and $d$ such that $a \leq c<x<d \leq b$ and $s(d) \leq h(c)$, belong to $L^{r, \infty}(w)$.

Let us observe that if $g \equiv 1$, we get that $\Phi_{1} \leq \Phi_{2}$. Then, in this case, the weighted weak-type inequality (i) is equivalent to $\Phi_{2} \in L^{r, \infty}(w)$. On the other hand, if $K \equiv 1$, then $\Phi_{1}=\Phi_{2}$ and we recover [1, Theorem 1.9].

To prove the theorem we will use the following lemma (see [1, Lemma 1.4] for the proof).

LEMmA 2.2. Let $a$ and $b$ be real numbers such that $a<b$. Let $s, h:(a, b) \rightarrow \mathbb{R}$ be increasing and continuous functions such that $s(x) \leq h(x)$ for all $x \in(a, b)$. Let $\left\{\left(a_{j}, b_{j}\right)\right\}_{j}$ be the connected components of the open set $\Omega=\{x \in(a, b): s(x)<h(x)\}$. Then

(a) $\left(s\left(a_{j}\right), h\left(b_{j}\right)\right) \cap\left(s\left(a_{i}\right), h\left(b_{i}\right)\right)=\varnothing$ for all $j \neq i$,

(b) for every $j$ there exists a (finite or infinite) sequence $\left\{m_{k}^{j}\right\}$ of real numbers such that:

(i) $a_{j} \leq m_{k}^{j}<m_{k+1}^{j} \leq b_{j}$ for all $k$ and $j$;

(ii) $\left(a_{j}, b_{j}\right)=\bigcup_{k}\left(m_{k}^{j}, m_{k+1}^{j}\right)$ a.e. for all $j$;

(iii) $s\left(m_{k+1}^{j}\right) \leq h\left(m_{k}^{j}\right)$ for all $k$ and $j$ and $s\left(m_{k+1}^{j}\right)=h\left(m_{k}^{j}\right)$ if $a_{j}<m_{k}^{j}<m_{k+1}^{j}<b_{j}$.

Proof of Theorem 2.1. (i) $\Rightarrow$ (ii). First, we will prove that $\Phi_{1} \in L^{r, \infty}(w)$, that is, we will prove that

$$
\sup _{\lambda>0} \lambda\left[w\left(\left\{x \in(a, b): \Phi_{1}(x)>\lambda\right\}\right)\right]^{1 / r}<\infty .
$$

Let $\lambda>0$ and $S_{\lambda}=\left\{x \in(a, b): \Phi_{1}(x)>\lambda\right\}$. For every $z \in S_{\lambda}$ there exist $\bar{c}_{z}$, $c_{z}$, and $d_{z}$, with $a \leq \bar{c}_{z} \leq c_{z}<z<d_{z} \leq b$ such that $s\left(d_{z}\right) \leq h\left(\bar{c}_{z}\right)$ and

$$
\lambda<\inf _{t \in\left(c_{z}, d_{z}\right)}\left[g(t) K\left(t, h\left(\bar{c}_{z}\right)\right)\right]\left(\int_{c_{z}}^{d_{z}} w\right)^{1 / p}\left(\int_{s\left(d_{z}\right)}^{h\left(\bar{c}_{z}\right)} v^{1-p^{\prime}}\right)^{1 / p^{\prime}} .
$$


Let $\mathscr{K} \subset S_{\lambda}$ be a compact set. Then there exist $\left(c_{z_{1}}, d_{z_{1}}\right), \ldots,\left(c_{z_{k}}, d_{z_{k}}\right)$ which cover $\mathscr{K}$. We may assume without loss of generality that $\sum_{j=1}^{k} \chi_{\left(c_{z_{j}}, d_{z_{j}}\right)} \leq 2 \chi_{\bigcup_{j=1}^{k}\left(c_{z_{j}}, d_{z_{j}}\right)}$. Let $f:(s(a), h(b)) \rightarrow$ $\mathbb{R}$ defined by

$$
f(y)=\left(\sum_{j=1}^{k} \frac{v^{-p^{\prime}}(y) \chi_{\left(s\left(d_{z_{j}}\right) h\left(\bar{c}_{z_{j}}\right)\right)}(y)}{\left(\inf _{t \in\left(c_{z_{j}}, d_{z_{j}}\right)}\left[g(t) K\left(t, h\left(\bar{c}_{z_{j}}\right)\right)\right] \int_{s\left(d_{z_{j}}\right)}^{h\left(\bar{c}_{z_{j}}\right)} v^{1-p^{\prime}}\right)^{p}}\right)^{1 / p} .
$$

If $z \in\left(c_{z_{j}}, d_{z_{j}}\right)$, then $\left(s\left(d_{z_{j}}\right), h\left(\bar{c}_{z_{j}}\right)\right) \subset(s(z), h(z))$ and since $K(z, y)$ is decreasing in $y$, we get that

$$
T f(z) \geq g(z) \int_{s\left(d_{z_{j}}\right)}^{h\left(\bar{c}_{z_{j}}\right)} K(z, y) f(y) d y \geq g(z) K\left(z, h\left(\bar{c}_{z_{j}}\right)\right) \int_{s\left(d_{z_{j}}\right)}^{h\left(\bar{c}_{z_{j}}\right)} f(y) d y \geq 1 .
$$

Therefore, $\bigcup_{j=1}^{k}\left(c_{z_{j}}, d_{z_{j}}\right) \subset\{x \in(a, b): T f(x) \geq 1\}$. Applying the weighted weak-type inequality and (2.5) we obtain

$$
\begin{aligned}
\int_{\bigcup_{j=1}^{k}\left(c_{z_{j}}, d_{z_{j}}\right)} w & \leq C\left(\sum_{j=1}^{k} \frac{\int_{s\left(d_{z_{j}}\right)}^{h\left(\bar{z}_{j}\right)} v^{1-p^{\prime}}}{\left(\inf _{t \in\left(c_{z_{j}}, d_{z_{j}}\right)}\left[g(t) K\left(t, h\left(\bar{c}_{z_{j}}\right)\right)\right] \int_{s\left(d_{z_{j}}\right)}^{h\left(\bar{z}_{z_{j}}\right)} v^{1-p^{\prime}}\right)^{p}}\right)^{q / p} \\
& =C\left(\sum_{j=1}^{k} \frac{1}{\inf _{t \in\left(c_{z_{j}}, d_{z_{j}}\right)}\left[g(t) K\left(t, h\left(\bar{c}_{z_{j}}\right)\right)\right]^{p}\left(\int_{s\left(d_{z_{j}}\right)}^{h\left(\bar{c}_{z_{j}}\right)} v^{1-p^{\prime}}\right)^{p-1}}\right)^{q / p} \\
& \leq \frac{C}{\lambda q}\left(\sum_{j=1}^{k} \int_{c_{z_{j} j}}^{d_{z_{j}}} w\right)^{q / p} \\
& \leq \frac{C}{\lambda q}\left(\int_{\bigcup_{j=1}^{k}\left(c_{z_{j}}, d_{z_{j}}\right)} w\right)^{q / p} .
\end{aligned}
$$

The last inequality implies that $\lambda\left(\int_{\mathscr{K}} w\right)^{1 / r} \leq C$ for any compact set $\mathscr{K} \subset S_{\lambda}$ which implies (2.4). The proof of (2.4) for the function $\Phi_{2}$ follows in a similar way applying $(i)$ to the function

$$
f(y)=\left(\sum_{j=1}^{k} \frac{K^{p^{\prime}}\left(c_{z_{j}}, y\right) v^{-p^{\prime}}(y) \chi_{\left(s\left(d_{z_{j}}\right), h\left(c_{z_{j}}\right)\right)}(y)}{\left(\inf _{t \in\left(c_{z_{j}}, d_{z_{j}}\right)} g(t) \int_{s\left(d_{z_{j}}\right)}^{h\left(c_{z_{j}}\right)} K^{p^{\prime}}\left(c_{z_{j}}, t\right) v^{1-p^{\prime}}(t) d t\right)^{p}}\right)^{1 / p} .
$$

(ii) $\Rightarrow$ (i). Let $\left\{a^{N}\right\}_{N=1}^{\infty}$ and $\left\{b^{N}\right\}_{N=1}^{\infty}$ be sequences in $(a, b)$ such that

$$
\lim _{N \rightarrow \infty} a^{N}=a, \quad \lim _{N \rightarrow \infty} b^{N}=b .
$$


A. L. Bernardis et al. 5

In order to prove (i) it will suffice to show that

$$
w\left(\left\{x \in\left(a^{N}, b^{N}\right): T f(x)>\lambda\right\}\right) \leq \frac{C}{\lambda q}
$$

for all nonnegative function $f$ bounded with compact support such that $\int_{s(a)}^{h(b)} f^{p} v=1$ and with a constant $C$ independent of $N, \lambda$, and $f$.

Let us fix $N \in \mathbb{N}$. Observe that if $O_{\lambda}=\left\{x \in\left(a^{N}, b^{N}\right): T f(x)>\lambda\right\}$ and $U=\{x \in(a, b)$ : $\left.\Phi_{1}(x) \leq \lambda^{q / r}, \Phi_{2}(x) \leq \lambda^{q / r}\right\}$, then

$$
\begin{aligned}
w\left(O_{\lambda}\right) \leq & w\left(O_{\lambda} \cap U\right)+w\left(\left\{x \in(a, b): \Phi_{1}(x)>\lambda^{q / r}\right\}\right) \\
& +w\left(\left\{x \in(a, b): \Phi_{2}(x)>\lambda^{q / r}\right\}\right) \\
\leq & w\left(O_{\lambda} \cap U\right)+\frac{\left\|\Phi_{1}\right\|_{r, \infty ; w}^{r}}{\lambda^{q}}+\frac{\left\|\Phi_{2}\right\|_{r, \infty, ; w}^{r}}{\lambda^{q}} .
\end{aligned}
$$

Therefore, the implication will be proved if we establish that $w\left(O_{\lambda} \cap U\right) \leq C / \lambda^{q}$. Let $\left(a_{j}, b_{j}\right)$ and $\left\{m_{k}^{j}\right\}$ be the sequences given by the lemma for the set $\Omega_{N}=\left\{x \in\left(a^{N}, b^{N}\right)\right.$ : $s(x)<h(x)\}$. Then, for fixed $j$,

$$
w\left(O_{\lambda} \cap U \cap\left(a_{j}, b_{j}\right)\right)=\sum_{k} w\left(O_{\lambda} \cap U \cap\left(m_{k}^{j}, m_{k+1}^{j}\right)\right) .
$$

If $x \in\left(m_{k}^{j}, m_{k+1}^{j}\right)$, since $s\left(m_{k+1}^{j}\right) \leq h\left(m_{k}^{j}\right)$, we get that

$$
\begin{aligned}
T f(x)= & g(x) \int_{s(x)}^{s\left(m_{k+1}^{j}\right)} K(x, y) f(y) d y+g(x) \int_{s\left(m_{k+1}^{j}\right)}^{h\left(m_{k}^{j}\right)} K(x, y) f(y) d y \\
& +g(x) \int_{h\left(m_{k}^{j}\right)}^{h(x)} K(x, y) f(y) d y=T_{j, k}^{1} f(x)+T_{j, k}^{2} f(x)+T_{j, k}^{3} f(x) .
\end{aligned}
$$

It is clear that

$$
w\left(O_{\lambda} \cap U \cap\left(m_{k}^{j}, m_{k+1}^{j}\right)\right) \leq w\left(E^{1}\right)+w\left(E^{2}\right)+w\left(E^{3}\right),
$$

where $E^{\ell}=\left\{x \in\left(m_{k}^{j}, m_{k+1}^{j}\right) \cap U: T_{j, k}^{\ell} f(x)>\lambda / 3\right\}, \ell=1,2,3$.

First, notice that the property (iii) of the kernel $K$ implies

$$
K(x, y) \leq D\left[K\left(x, h\left(m_{k}^{j}\right)\right)+K\left(m_{k}^{j}, y\right)\right]
$$

for $x \in\left(m_{k}^{j}, m_{k+1}^{j}\right)$ and $y \in\left(s\left(m_{k+1}^{j}\right), h\left(m_{k}^{j}\right)\right)$. 
In order to estimate $w\left(E^{1}\right)$ let us observe that

$$
\begin{aligned}
T_{j, k}^{1} f(x) \leq & D g(x) K\left(x, h\left(m_{k}^{j}\right)\right) \int_{s(x)}^{s\left(m_{k+1}^{j}\right)} f(y) d y \\
& +D g(x) \int_{s(x)}^{s\left(m_{k+1}^{j}\right)} K\left(m_{k}^{j}, y\right) f(y) d y=D T_{j, k}^{1,1} f(x)+D T_{j, k}^{1,2} f(x) .
\end{aligned}
$$

Then, $w\left(E^{1}\right) \leq w\left(E^{1,1}\right)+w\left(E^{1,2}\right)$, where

$$
E^{1, \ell}=\left\{x \in\left(m_{k}^{j}, m_{k+1}^{j}\right) \cap U: T_{j, k}^{1, \ell} f(x)>\frac{\lambda}{6 D}\right\}, \quad \ell=1,2
$$

Let us select an increasing sequence $\left\{x_{i}\right\}_{i}, x_{i} \in\left(m_{k}^{j}, m_{k+1}^{j}\right)$, such that $x_{0}=m_{k}^{j}$ and

$$
\int_{s\left(x_{i}\right)}^{s\left(m_{k+1}^{j}\right)} f=\int_{s\left(x_{i-1}\right)}^{s\left(x_{i}\right)} f .
$$

Let $E_{i}^{1,1}=E^{1,1} \cap\left(x_{i}, x_{i+1}\right), \alpha_{i}^{1}=\inf E_{i}^{1,1}$, and $\beta_{i}^{1}=\sup E_{i}^{1,1}$. If $E_{i}^{1,1} \neq \varnothing$, let $t \in E_{i}^{1,1}$. Using the property of the sequence $\left\{x_{i}\right\}_{i}$ we have

$$
\frac{\lambda}{6 D} \leq 4 g(t) K\left(t, h\left(m_{k}^{j}\right)\right) \int_{s\left(x_{i+1}\right)}^{s\left(x_{i+2}\right)} f .
$$

Now, by using (1.3) and Hölder inequality we get

$$
\frac{\lambda}{6 D} \leq 4 \inf _{t \in\left(\alpha_{i}^{1}, \beta_{i}^{1}\right)}\left[g(t) K\left(t, h\left(m_{k}^{j}\right)\right)\right]\left(\int_{s\left(x_{i+1}\right)}^{s\left(x_{i+2}\right)} v^{1-p^{\prime}}\right)^{1 / p^{\prime}}\left(\int_{s\left(x_{i+1}\right)}^{s\left(x_{i+2}\right)} f^{p} v\right)^{1 / p} .
$$

Now, multiplying by $\left(\int_{\alpha_{i}^{1}}^{\beta_{i}^{1}} w\right)^{1 / p}$ and using the inequalities $s\left(\beta_{i}^{1}\right) \leq s\left(x_{i+1}\right)$ and $s\left(x_{i+2}\right) \leq$ $s\left(m_{k+1}^{j}\right) \leq h\left(m_{k}^{j}\right)$ we get that

$$
\frac{\lambda}{6 D}\left(\int_{\alpha_{i}^{1}}^{\beta_{i}^{1}} w\right)^{1 / p} \leq 4 \Phi_{1}(x)\left(\int_{s\left(x_{i+1}\right)}^{s\left(x_{i+2}\right)} f^{p} v\right)^{1 / p} \leq 4 \lambda^{q / r}\left(\int_{s\left(x_{i+1}\right)}^{s\left(x_{i+2}\right)} f^{p} v\right)^{1 / p},
$$

where $x$ is any element of $E_{i}^{1,1}$; and summing up in $i$ we obtain

$$
w\left(E^{1,1}\right) \leq \frac{C}{\lambda^{q}} \int_{s\left(m_{k}^{j}\right)}^{s\left(m_{k+1}^{j}\right)} f^{p} v
$$

To estimate $w\left(E^{1,2}\right)$, we select an increasing sequence $\left\{z_{i}\right\}_{i}, z_{i} \in\left(m_{k}^{j}, m_{k+1}^{j}\right)$ such that $z_{0}=$ $m_{k}^{j}$ and

$$
\int_{s\left(z_{i}\right)}^{s\left(m_{k+1}^{j}\right)} K\left(m_{k}^{j}, y\right) f(y) d y=\int_{s\left(z_{i-1}\right)}^{s\left(z_{i}\right)} K\left(m_{k}^{j}, y\right) f(y) d y
$$


As before, let $E_{i}^{1,2}=E^{1,2} \cap\left(z_{i}, z_{i+1}\right), \alpha_{i}^{2}=\inf E_{i}^{1,2}$, and $\beta_{i}^{2}=\sup E_{i}^{1,2}$. If $E_{i}^{1,2} \neq \varnothing$, then Hölder inequality and (1.2) give

$$
\frac{\lambda}{6 D} \leq 4 \inf _{t \in\left(\alpha_{i}^{2}, \beta_{i}^{2}\right)} g(t)\left(\int_{s\left(z_{i+1}\right)}^{s\left(z_{i+2}\right)} K^{p^{\prime}}\left(m_{k}^{j}, t\right) v^{1-p^{\prime}}(t) d t\right)^{1 / p^{\prime}}\left(\int_{s\left(z_{i+1}\right)}^{s\left(z_{i+2}\right)} f^{p} v\right)^{1 / p} .
$$

Notice that $s\left(\beta_{i}^{2}\right) \leq s\left(z_{i+1}\right), m_{k}^{j} \leq \alpha_{i}^{2}$, and $s\left(z_{i+2}\right) \leq s\left(m_{k+1}^{j}\right) \leq h\left(m_{k}^{j}\right) \leq h\left(\alpha_{i}^{2}\right)$. Then multiplying by $\left(\int_{\alpha_{i}^{2}}^{\beta_{i}^{2}} w\right)^{1 / p}$ both members of the above inequality we get

$$
\frac{\lambda}{6 D}\left(\int_{\alpha_{i}^{2}}^{\beta_{i}^{2}} w\right)^{1 / p} \leq 4 \Phi_{2}(x)\left(\int_{s\left(z_{i+1}\right)}^{s\left(z_{i+2}\right)} f^{p} v\right)^{1 / p} \leq 4 \lambda^{q / r}\left(\int_{s\left(z_{i+1}\right)}^{s\left(z_{i+2}\right)} f^{p} v\right)^{1 / p}
$$

where $x$ is any element of $E_{i}^{1,2}$. Now, summing up in $i$ and putting together with (2.23) we obtain

$$
w\left(E^{1}\right) \leq \frac{C}{\lambda q} \int_{s\left(m_{k}^{j}\right)}^{s\left(m_{k+1}^{j}\right)} f^{p} v
$$

To estimate $w\left(E^{2}\right)$ we proceed in a similar way. In fact, by using (2.16) we get that

$$
\begin{aligned}
T_{j, k}^{2} f(x) \leq & D g(x) K\left(x, h\left(m_{k}^{j}\right)\right) \int_{s\left(m_{k+1}^{j}\right)}^{h\left(m_{k}^{j}\right)} f(y) d y \\
& +D g(x) \int_{s\left(m_{k+1}^{j}\right)}^{h\left(m_{k}^{j}\right)} K\left(m_{k}^{j}, y\right) f(y) d y=D T_{j, k}^{2,1} f(x)+D T_{j, k}^{2,2} f(x),
\end{aligned}
$$

which implies that $w\left(E^{2}\right) \leq w\left(E^{2,1}\right)+w\left(E^{2,2}\right)$, where the sets $E^{2, \ell}, \ell=1,2$ are defined as the sets $E^{1, \ell}$ with $T_{j, k}^{2, \ell} f$ instead of $T_{j, k}^{1, \ell} f$. Now, the estimates of $w\left(E^{2,1}\right)$ and $w\left(E^{2,2}\right)$ follow as in the previous cases obtaining

$$
w\left(E^{2}\right) \leq \frac{C}{\lambda^{q}} \int_{s\left(m_{k+1}^{j}\right)}^{h\left(m_{k}^{j}\right)} f^{p} v .
$$

Actually, the estimations are easier because we do not need to split the sets $E^{2, \ell}$. For the estimation of $w\left(E^{3}\right)$ let us define the function

$$
H(x)=\int_{h\left(m_{k}^{j}\right)}^{h(x)} K(x, y) f(y) d y
$$

Since $h$ is continuous and $K$ is continuous in the first variable, we may select a decreasing 
sequence $\left\{x_{i}\right\}_{i}$ in $\left(m_{k}^{j}, m_{k+1}^{j}\right)$ such that $x_{0}=m_{k+1}^{j}$ and $H\left(x_{i}\right)=\int_{h\left(m_{k}^{j}\right)}^{h\left(x_{i}\right)} K\left(x_{i}, y\right) f(y) d y=$ $(D+1)^{-i} H\left(m_{k+1}^{j}\right)$. We claim that

$$
H\left(x_{i}\right) \leq(D+1)^{4}\left(K\left(x_{i+2}, h\left(x_{i+3}\right)\right) \int_{h\left(m_{k}^{j}\right)}^{h\left(x_{i+3}\right)} f(y) d y+\int_{h\left(x_{i+3}\right)}^{h\left(x_{i+2}\right)} K\left(x_{i+2}, y\right) f(y) d y\right) .
$$

In fact, first notice that

$$
\begin{aligned}
H\left(x_{i}\right) & =(D+1)^{2} \int_{h\left(m_{k}^{j}\right)}^{h\left(x_{i+2}\right)} K\left(x_{i+2}, y\right) f(y) d y \\
& =(D+1)^{2}\left[\int_{h\left(m_{k}^{j}\right)}^{h\left(x_{i+3}\right)} K\left(x_{i+2}, y\right) f(y) d y+\int_{h\left(x_{i+3}\right)}^{h\left(x_{i+2}\right)} K\left(x_{i+2}, y\right) f(y) d y\right] .
\end{aligned}
$$

Now, applying property (iii) of $K$ we get that

$$
\begin{aligned}
H\left(x_{i}\right) \leq & D(D+1)^{2}\left[K\left(x_{i+2}, h\left(x_{i+3}\right)\right) \int_{h\left(m_{k}^{j}\right)}^{h\left(x_{i+3}\right)} f(y) d y+\int_{h\left(m_{k}^{j}\right)}^{h\left(x_{i+3}\right)} K\left(x_{i+3}, y\right) f(y) d y\right] \\
& +(D+1)^{2} \int_{h\left(x_{i+3}\right)}^{h\left(x_{i+2}\right)} K\left(x_{i+2}, y\right) f(y) d y \\
\leq & (D+1)^{3}\left[K\left(x_{i+2}, h\left(x_{i+3}\right)\right) \int_{h\left(m_{k}^{j}\right)}^{h\left(x_{i+3}\right)} f(y) d y+\int_{h\left(x_{i+3}\right)}^{h\left(x_{i+2}\right)} K\left(x_{i+2}, y\right) f(y) d y\right] \\
& +\frac{D}{D+1} H\left(x_{i}\right),
\end{aligned}
$$

and the claim follows. Now, we have

$$
w\left(E^{3}\right) \leq \sum_{i \geq 0}\left[w\left(E_{i}^{3,1}\right)+w\left(E_{i}^{3,2}\right)\right]
$$

where

$$
\begin{gathered}
E_{i}^{3,1}=\left\{x \in\left(x_{i+1}, x_{i}\right) \cap U: g(x) K\left(x_{i+2}, h\left(x_{i+3}\right)\right) \int_{h\left(m_{k}^{j}\right)}^{h\left(x_{i+3}\right)} f(y) d y>\frac{\lambda}{6(D+1)^{4}}\right\}, \\
E_{i}^{3,2}=\left\{x \in\left(x_{i+1}, x_{i}\right) \cap U: g(x) \int_{h\left(x_{i+3}\right)}^{h\left(x_{i+2}\right)} K\left(x_{i+2}, y\right) f(y) d y>\frac{\lambda}{6(D+1)^{4}}\right\} .
\end{gathered}
$$

Working as in previous cases we have

$$
\sum_{i \geq 0} w\left(E_{i}^{3,2}\right) \leq \frac{C}{\lambda^{q}} \int_{h\left(m_{k}^{j}\right)}^{h\left(m_{k+1}^{j}\right)} f^{p} v
$$


A. L. Bernardis et al. 9

In order to estimate $\sum_{i \geq 0} w\left(E_{i}^{3,1}\right)$ we will apply the ideas of [4, Lemma 1]. Let $\left\{u_{s}^{\prime}\right\}$ be the decreasing sequence in $\left(m_{k}^{j}, m_{k+1}^{j}\right)$ defined by $u_{0}^{\prime}=m_{k+1}^{j}$ and

$$
\int_{h\left(m_{k}^{j}\right)}^{h\left(u_{s}^{\prime}\right)} f=2^{-s} \int_{h\left(m_{k}^{j}\right)}^{h\left(m_{k+1}^{j}\right)} f
$$

and let $\left\{u_{n}\right\}$ be the subsequence of $\left\{u_{s}^{\prime}\right\}$ defined by $u_{0}=u_{0}^{\prime}$ and if $\left[u_{s+1}^{\prime}, u_{s}^{\prime}\right) \cap\left\{x_{i}\right\}=\varnothing$, then we delete the term $u_{s+1}^{\prime}$ of $\left\{u_{s}^{\prime}\right\}$. Let $\widetilde{E}_{n}^{3,1}=\bigcup_{\left\{i \geq 0: u_{n+1} \leq x_{i+3}<u_{n}\right\}} E_{i}^{3,1}, \widetilde{\alpha}_{n}=\inf \widetilde{E}_{n}^{3,1}$, and $\widetilde{\beta}_{n}=\sup \widetilde{E}_{n}^{3,1}$. If $u_{s+1}^{\prime}=u_{n+1} \leq x_{i+3}<u_{n}$, by the construction of the sequences we get that $x_{i+3} \leq u_{s}^{\prime}$ and $u_{n+2} \leq u_{s+2}^{\prime}$, then

$$
\int_{h\left(m_{k}^{j}\right)}^{h\left(x_{i+3}\right)} f \leq \int_{h\left(m_{k}^{j}\right)}^{h\left(u_{s}^{\prime}\right)} f=4 \int_{h\left(u_{s+2}^{\prime}\right)}^{h\left(u_{s+1}^{\prime}\right)} f \leq 4 \int_{h\left(u_{n+2}\right)}^{h\left(u_{n+1}\right)} f .
$$

Let us assume that $\widetilde{E}_{n}^{3,1} \neq \varnothing$. By the above inequalities and the monotonicity of $K$ we have for all $t \in \widetilde{E}_{n}^{3,1}$,

$$
\frac{\lambda}{6(D+1)^{4}} \leq 4 g(t) K\left(t, h\left(x_{i+3}\right)\right) \int_{h\left(u_{s+2}^{\prime}\right)}^{h\left(u_{s+1}^{\prime}\right)} f \leq 4 g(t) K\left(t, h\left(u_{n+1}\right)\right) \int_{h\left(u_{n+2}\right)}^{h\left(u_{n+1}\right)} f .
$$

Now, multiplying by $\left(\int_{\widetilde{\alpha}_{n}}^{\widetilde{\beta}_{n}} w\right)^{1 / p}$, applying Hölder inequality, and using that $s\left(\widetilde{\beta}_{n}\right) \leq h\left(u_{n+2}\right)$ we get that

$$
\frac{\lambda}{6(D+1)^{4}}\left(\int_{\widetilde{\alpha}_{n}}^{\tilde{\beta}_{n}} w\right)^{1 / p} \leq 4 \Phi_{1}(x)\left(\int_{h\left(u_{n+2}\right)}^{h\left(u_{n+1}\right)} f^{p} v\right)^{1 / p} \leq 4 \lambda^{q / r}\left(\int_{h\left(u_{n+2}\right)}^{h\left(u_{n+1}\right)} f^{p} v\right)^{1 / p}
$$

where $x$ is any point in $\widetilde{E}_{n}^{3,1}$. Then

$$
\begin{aligned}
\sum_{i \geq 0} w\left(E_{i}^{3,1}\right) & =\sum_{n} \sum_{\left\{i \geq 0: u_{n+1} \leq x_{i+3}<u_{n}\right\}} w\left(E_{i}^{3,1}\right) \\
& \leq \sum_{n} w\left(\widetilde{E}_{n}^{3,1}\right) \leq \sum_{n} \int_{\widetilde{\alpha}_{n}}^{\widetilde{\beta}_{n}} w \\
& \leq \frac{C}{\lambda^{q}} \sum_{n} \int_{h\left(u_{n+2}\right)}^{h\left(u_{n+1}\right)} f^{p} v \leq \frac{C}{\lambda^{q}} \int_{h\left(m_{k}^{j}\right)}^{h\left(m_{k+1}^{j}\right)} f^{p} v .
\end{aligned}
$$

Putting together the estimations of $w\left(E^{1}\right), w\left(E^{2}\right)$, and $w\left(E^{3}\right)$ we have

$$
w\left(O_{\lambda} \cap U \cap\left(m_{k}^{j}, m_{k+1}^{j}\right)\right) \leq \frac{C}{\lambda^{q}} \int_{s\left(m_{k}^{j}\right)}^{h\left(m_{k+1}^{j}\right)} f^{p} v .
$$


Summing up in $k$ in the above inequality and by (2.13) we get that

$$
w\left(O_{\lambda} \cap U \cap\left(a_{j}, b_{j}\right)\right) \leq \frac{C}{\lambda^{q}} \int_{s\left(a_{j}\right)}^{h\left(b_{j}\right)} f^{p} v .
$$

Keeping in mind the lemma and summing up in $j$ we obtain the desired inequality.

\section{Acknowledgments}

This research has been partially supported by Spanish goverment Grant MTM2005-8350C03-02. The first author was supported in part by CAI+D-UNL and CONICET. The second and third authors were supported by Junta de Andalucía Grant FQM 354.

\section{References}

[1] A. L. Bernardis, F. J. Martín-Reyes, and P. Ortega Salvador, Weighted inequalities for HardySteklov operators, to appear in Canadian Journal of Mathematics.

[2] T. Chen and G. Sinnamon, Generalized Hardy operators and normalizing measures, Journal of Inequalities and Applications 7 (2002), no. 6, 829-866.

[3] A. Gogatishvili and J. Lang, The generalized Hardy operator with kernel and variable integral limits in Banach function spaces, Journal of Inequalities and Applications 4 (1999), no. 1, 1-16.

[4] Q. Lai, Weighted modular inequalities for Hardy type operators, Proceedings of the London Mathematical Society. Third Series 79 (1999), no. 3, 649-672.

[5] F. J. Martín-Reyes and P. Ortega Salvador, On weighted weak type inequalities for modified Hardy operators, Proceedings of the American Mathematical Society 126 (1998), no. 6, 1739-1746.

[6] D. V. Prokhorov, Weighted estimates for Riemann-Liouville operators with variable limits, Siberian Mathematical Journal 44 (2003), no. 6, 1049-1060.

A. L. Bernardis: IMAL-CONICET, Güemes 3450, Santa Fe 3000, Argentina

E-mail address: bernard@ceride.gov.ar

F. J. Martín-Reyes: Departamento de Análisis Matemático, Facultad de Ciencias, Universidad de Málaga, 29071 Málaga, Spain

E-mail address: martin_reyes@uma.es

P. Ortega Salvador: Departamento de Análisis Matemático, Facultad de Ciencias, Universidad de Málaga, 29071 Málaga, Spain

E-mail address: ortega@anamat.cie.uma.es 